\title{
Implementing Radial Motion to the Booster Simulation
}

\author{
Xi Yang
}

April 5, 2007

\section{Motivation}

It's a puzzle that high intensity beams prefer a particular radial motion during transition in the Booster, and the result of removing such a radial motion is to increase the transition loss. In order to understand this observation, the radial motion should be taken into account in the longitudinal simulation.

\section{Method}

The magnets are ramped from $400 \mathrm{MeV}$ to $8 \mathrm{GeV}$ with a repetition rate of $15 \mathrm{~Hz}$, and it defines the accelerating ramp. Here, $400 \mathrm{MeV}$ and $8 \mathrm{GeV}$ are the kinetic energies of a proton at injection and extraction. A virtual particle which follows the central orbit all the time is defined as Reference Particle, and it's used as a clock to track the accelerating ramp; a virtual particle which follows the radial offset curve in an accelerating cycle is defined as Synchronous Particle, and it defines the synchrotron oscillation center. At the moment Synchronous Particle has less energy than Reference Particle, the radial offset is negative, and the beam position is negative horizontally, and vice versa. From the radial offset $(R)$ and the dispersion $(D)$ where the radial-offset detector is, the momentum deviation of Synchronous Particle relative to Reference Particle is calculated using equation (1).

$$
X_{s}(t)=R(t)=D(t) \cdot \frac{P_{s}-P_{0}}{P_{0}}=D(t) \cdot \delta_{s}(t)
$$

Here, $P_{s}$ and $P_{0}$ are momenta of Synchronous Particle and Reference Particle. Afterwards, the slippage factor of Synchronous Particle $\eta\left(\delta_{s}\right)$ is calculated using equation (2).[1]

$$
\eta\left(\delta_{s}\right) \approx \alpha_{0}-\frac{1}{\gamma_{0}^{2}}+\alpha_{0} \cdot\left(\alpha_{1}+1.5 \cdot \beta_{0}^{2}\right) \cdot \delta_{\mathrm{s}}+\left[\alpha_{0} \cdot \alpha_{2}+\frac{\left(1-5 \cdot \beta_{0}^{2}\right) \cdot \beta_{0}^{2}}{2 \cdot \gamma_{0}^{2}}\right] \cdot \delta_{s}^{2}
$$


Here, $\alpha_{0}, \alpha_{1}$, and $\alpha_{2}$ are $0^{\text {th }}, 1^{\text {st }}$, and $2^{\text {nd }}$ order momentum compaction factors, $\gamma_{0}$ and $\beta_{0}$ are the Lorentz relativistic factors of Reference Particle. Finally, at time $t$, the revolution time of Synchronous Particle, $t_{s}$, is calculated using equation (3).

$$
\frac{\Delta t}{t_{0}}=\frac{t_{s}-t_{0}}{t_{0}}=\eta\left(\delta_{s}\right) \cdot \delta_{s}(t)
$$

Here, $t_{0}$ is the revolution time of Reference Particle. The time taken by the beam to complete a turn at time $t$ in a cycle is determined by $t_{s}$, and $t_{0}$ serves as the perturbation expansion center.

During the present turn, the amount of energy gain $\Delta E_{s}(t)$ needed for the beam to follow the accelerating ramp is calculated using equation (4). The beam is injected at $t=0.0 \mathrm{~s}$.

$$
\begin{aligned}
& P_{0}(t)=P_{i n j}+0.5 \cdot\left(P_{e x t}-P_{i n j}\right) \cdot\left[1-\cos \left(2 \pi f_{r e p} \cdot t\right)\right] \\
& P_{s}(t)=P_{0}(t) \cdot\left(1+\delta_{s}(t)\right) \\
& P_{0}\left(t+t_{s}\right)=P_{i n j}+0.5 \cdot\left(P_{e x t}-P_{i n j}\right) \cdot\left[1-\cos \left(2 \pi f_{r e p} \cdot\left(t+t_{s}\right)\right)\right] \\
& P_{s}\left(t+t_{s}\right)=P_{0}\left(t+t_{s}\right) \cdot\left(1+\delta_{s}\left(t+t_{s}\right)\right) \\
& E_{s}(t)=\sqrt{\left(P_{s}(t)\right)^{2}+E_{r e s}^{2}} \\
& E_{s}\left(t+t_{s}\right)=\sqrt{\left(P_{s}\left(t+t_{s}\right)\right)^{2}+E_{r e s}^{2}} \\
& \Delta E_{s}(t)=E_{s}\left(t+t_{s}\right)-E_{s}(t)
\end{aligned}
$$

Here, " $P_{i n j}=0.954263 \mathrm{GeV} / \mathrm{c}$ " and " $P_{e x t}=8.88889 \mathrm{GeV} / \mathrm{c}$ " are the momenta at injection and extraction respectively, " $f_{\text {rep }}=15 \mathrm{~Hz}$ " is the repetition rate, " $E_{\text {res }}=0.93827231 \mathrm{GeV}$ " is the rest energy of a proton.

The rf voltage at time $t$ is $V(t)$, which is vector sum from all the rf stations. The synchronous phase $\varphi_{s}(t)$, which is the phase of Synchronous Particle relative to the rf waveform, is calculated using equation (5). The unit of $E_{s}(t)$ and $V(t)$ should be consistent, and if $E_{s}(t)$ is in unit of "GeV", $V(t)$ should be in unit of GV, etc. When the radial feedback is used, the phase shift required by the feedback system should be added to $\varphi_{s}(t)$ in equation $(5)$. 


$$
\varphi_{s}(t)=\sin ^{-1}\left(\frac{\Delta E_{s}(t)}{V(t)}\right)
$$

\section{Conclusion}

The above procedures have been implemented to Booster simulations in Fortran. All the calculations related to perturbation expansions use Reference Particle, and all the calculations related to the beam parameters use Synchronous Particle. The code also can be applied to other accelerators. The preferred radial motion by high intensity beams at transition is understood [2,3] and approved by numerical simulation since then.[4]

\section{Acknowledgements}

A. Drozhdin for allowing me to add the program to STRUCT and checking it with me. V. Lebedev, F. Ostiguy, and J. MacLachlan for useful discussions.

References:

- $\quad$ [1] J. Wei, Ph.D thesis (1990)

- [2] V. A. Lebedev, "RF voltage jump", Beams-doc-2690-v1.

- [3] X. Yang and W. Pellico, Beams-doc-2686-v1.

- [4] X. Yang, FERMILAB-FN-0797-AD. 